\title{
Role of Platelets in Glutamate Mediated Excitotoxicity: An Overview
}

\author{
Deepa $^{1,2}$, Arundhati Tiwari ${ }^{1}$, Rameshwar Nath Chaurasia ${ }^{2}$ and Debabrata Dash ${ }^{1^{*}}$ \\ ${ }^{1}$ Department of Biochemistry, Institute of Medical Sciences, Banaras Hindu University, Varanasi, India \\ ${ }^{2}$ Department of Neurology, Institute of Medical Sciences, Banaras Hindu University, Varanasi, India
}

*Corresponding author: Debabrata Dash, Department of Biochemistry, Institute of Medical Sciences, Banaras Hindu University, Varanasi, India, Tel: +91 9336910665, Fax: +91 542 2367568; E-mail: ddash.biochem@gmail.com

Received date: July 07, 2015; Accepted date: August 10, 2015; Published date: August 17, 2015

Copyright: (2015 Deepa et al. This is an open-access article distributed under the terms of the Creative Commons Attribution License, which permits unrestricted use, distribution, and reproduction in any medium, provided the original author and source are credited.

\begin{abstract}
Glutamate, the main excitatory neurotransmitter in central nervous system (CNS), binds primarily to two types of receptors on the post-synaptic membrane of neuronal cells, namely metabotropic receptors and ionotropic receptors. Metabotropic receptors are G-protein coupled receptors while ionotropic receptors are non-selective ligand-gated ion channels, which allow movement of cations like $\mathrm{Na}+, \mathrm{K}+$ and $\mathrm{Ca} 2+$. The three most studied ionic receptors are N-methyl-D-aspartate receptor (NMDAR), a-amino-3-hydroxy-5-methyl-4-isoxazolepropionic acid receptor (AMPAR), and Kainate receptor.

Glutamate synapse plays a pivotal role in many neurodegenerative disorders like Huntington disease, trauma, epilepsy, Alzheimer and Amyotrophic lateral sclerosis, and cerebral ischemia. Understanding the mechanism of these glutamate ligand-gated ion channels (LGIC) can assist in the development of therapy against ischemic stroke, a leading cause of death and disability worldwide.

This review focuses on how aggregated platelet microthrombi cross blood-brain barrier, reach neural parenchyma and release glutamate. Accumulated glutamate hyperstimulates glutamate LGIC, thus leading to neurotoxicity and apoptosis of neuronal cells.
\end{abstract}

\section{Introduction}

Stroke is one of the major challenges faced by almost all the developing countries. Stroke has great impact worldwide as it is one of the capital causes of death and affliction. WHO endeavored to define stroke as "Rapidly developing signs of focal (or global) disturbance of cerebral function, lasting more than 24 hours or leading to death, with no apparent cause other than that of vascular origin" [1].

According to the recent report submitted to $\mathrm{WHO}$, the rate of stroke is $41 / 100,000$ population in Nigeria and $316 / 100,000$ population in Dar-es-salaam. In Europe stroke incidence rate is 101.1-239.3/100,1000 men population and 63.0-158.7/100,000 women population [2]. The prevalence of stroke in India in recent years has been around $84-262 / 100,000$ in rural and $334-424 / 100,000$ population per year in urban areas [3].

Ischemic stroke becomes a higher risk, when platelet aggregates (primary injury) release glutamate and elevate amino acid concentration in the body fluid. The released glutamate interacts with glutamate receptors on endothelial cells of blood-brain barrier leading to breakdown of the barrier. Glutamate excitotoxicity is the final pathway that results in either clinical deficits or complete loss of the functions of neuronal cells (secondary injury).

\section{Parallelism between Platelet and Neuron}

Sharing the common embryonic origin i.e. ectodermic, platelets and neurons show a strong resemblance among themselves. To study neuronal molecular mechanism, the most significant model is platelet because it has morphological and functional similarities with neuron.
Serotonin transport mechanism is similar in platelet and neuron [4]. Other neurotransmitters like dopamine, gamma-aminobutyric acid (GABA), and glutamate are secreted by both the cells [5]. Glutamate receptors are present on neuronal as well as non-neuronal tissues like bone marrow, kidney, heart, pancreas and platelets [6].

Platelets have been used in understanding the mechanism of various neurological disorders like migraine, Parkinson, autism, schizophrenia and Alzheimer's [7]. Alzheimer is caused due to senile plaque formation in the brain by amyloid $\beta$ and $95 \%$ of circulating amyloid-precursor protein in blood is contributed by platelets. We have shown that when platelets are treated with $A \beta 25-35$, small GTPase RhoA gets activated and in turn activate platelets by modulating actomyosin, leading to cytoskeleton reorganization [8].

\section{Glutamate Receptor}

Glutamate is the main excitatory neurotransmitter in vertebrate CNS and the most abundant amino acid in diet. It plays critical role in many neurophysiologic functions like memory, learning, cognition, mood alteration and executive functioning. Glutamate has the postsynaptic effect on two broadly classified glutamate receptors, i.e., ionotropic and metabotropic receptors. The former are G proteincoupled receptors, which recruit second messengers such as diacylglycerol and cAMP to modify neuronal excitability. Ionotropic glutamate receptors are non-selective cationic ligand gated ion channels having significant role in excitatory signaling. Mainly three classes of receptors fall in this category viz. NMDAR, AMPAR, Kainate receptors, named after responsiveness and affinity to preferred agonists, N-methyl -D-aspartate, a amino-3-hydroxy-5-methyl-4- 
isoxazoepropionic acid, kainic acid, respectively. NMDAR is a major subfamily of glutamate receptors that favors $\mathrm{Ca} 2+$ inflow but also allows the passage of monovalent cations like $\mathrm{Na}+$ and $\mathrm{K}+$. Different subunits of NMDAR are expressed that include GluNR1, GluNR2A, GluNR2B, GluNR2C, GluNR2D, and GluNR3A. Two subunits of GluNR1 integrate with either same or different GluNR2 or rarely with GluNR3A subunit to form tetrameric complex. NMDAR needs ligands like NMDA or glutamate binding to GluNR2 subunit and co-ligands like glycine or D-serine binding to GluNR1 or GluNR3 subunit for its opening [9].

Another significant receptor is AMPA receptor, a heterotetramer complex composed of GluR1-R4 subunits. Intact receptor complex is $\mathrm{Ca} 2+$ impermeable ion channel, whereas absence of GluR2 subunit from the complex increases permeability for $\mathrm{Ca} 2+$ and $\mathrm{Zn} 2+$ by 2 to 3 folds. One of the calcium-activated cytosolic enzymes is protein kinase $c-\alpha(\mathrm{PKC} \alpha)$. Once activated in presence of $\mathrm{Ca} 2+, \mathrm{PKC} \alpha$ forms complex with Protein interacting with $\mathrm{C}$ kinase 1 (PICK1) and translocate to plasma membrane where it phosphorylates GluR2 subunit of AMPAR at serine 880 residue. Phosphorylation of GluR2 triggers its endocytosis, thus making AMPAR Ca2+ permeable [10].

\section{Primary and Secondary Neuronal Injury}

Platelet aggregation leads to blockage of arteries supplying to brain, thus depriving brain of oxygen and nutrients. It results in drop in energy charge in cells and neuronal cell death (primary injury) (Figure 1). Recently it has been demonstrated that during the course of primary injury platelet activation/aggregation amplification is regulated by NMDA receptors. Platelet aggregation is inhibited by antagonists of NMDA receptor like MK801 hydrogen maleate, 3, 5dimethyl -1-adanantamine hydrochloride (memantine), D-2-amino 5phosphopentanoic acid (AP5), while NMDAR agonists like Lglutamate, glycine, NMDA facilitate platelet activation [11].

It is hypothesized that platelets on aggregation release glutamate, which mediates excitotoxic brain injury and neuronal dysfunction. Glutamate is stored in platelet dense granules and is released when platelets form aggregates. Platelets express vesicular glutamate transporters (VGLUT), through which they release glutamate after formation of microthrombi [12].

Thrombin-evoked release of glutamate from platelets has been quantified. Platelets release glutamate within 60 seconds of thrombin treatment, raising glutamate concentration from $175 \mu \mathrm{M}$ to a maximum of $425 \mu \mathrm{M}$. Blood brain barrier becomes permeable after subarachnoid hemorrhage and delayed cerebral ischemia. It might be possible that when stimulated platelets form thrombus, glutamate released from them binds to and stimulates NMDAR expressed on endothelial cells. It leads to disintegration of blood-brain barrier and decamping of platelets into neuronal parenchyma, thus mediating glutamate excitotoxicity by exposing neuronal cells to glutamate. Neurotoxicity or secondary injury is caused when released glutamate binds to its ionotropic receptors like NMDA and AMPA on neuronal cells, which leads to disturbance in ionic homeostasis. Secondary injury to neurons can either be due to clinical deficits or neuronal programmed cell death (Figure 1). There is a direct relationship between microthrombosis and changed neuronal physiological properties. Such changes can result in difficulties with memory, consolidation, confusion and executive functions $[13,14]$.

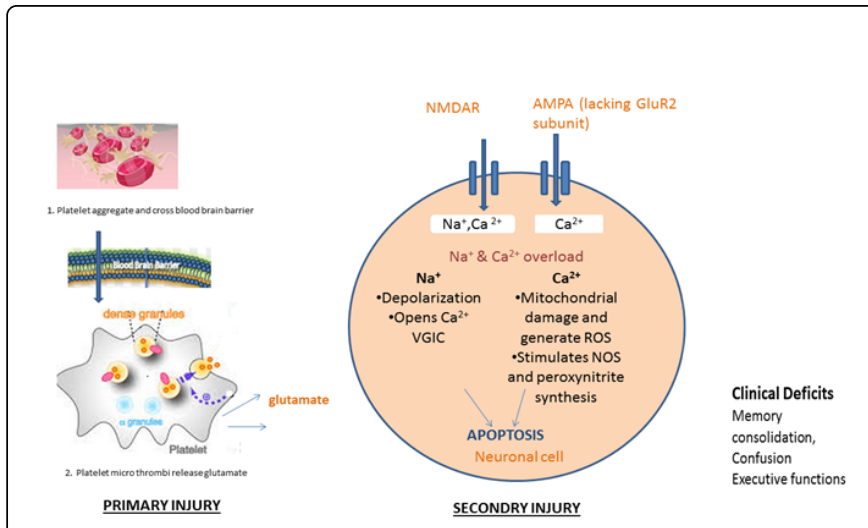

Figure 1: Primary injury is caused due to formation of microthrombi and crossing of blood-brain barrier. Microthrombi accumulate in neuronal parenchyma and release glutamate, which stimulates glutamate ionic receptors i.e. NMDAR and AMPAR. Secondary injury is caused due to over-stimulated glutamate ionic receptors, which disrupt ionic balance leading to two consequences: either apoptosis by triggering mitochondrial damage and forming reactive oxygen species or causing clinical deficits. (NMDAR, Nmethyl-D-aspartate receptor; AMPAR, a amino-3-hydroxy-5methyl-4-isoxazoepropionic acid receptor; VGIC, Voltage-gated ion channel; NOS, Nitric oxide synthase).

\section{Neurotoxicity Mediated by Excessive Glutamate Accumulation}

Hyperstimulation of glutamate LGIC disrupts the ionic homeostasis. A transporter protein, $\mathrm{Na}+-\mathrm{K}+-\mathrm{Cl}-$ (NKCC1) cotransporter 1, of neuron is stimulated in presence of glutamate and disrupts ion homeostasis in ischemic condition. NKCC1 allows the entry of $\mathrm{Na}+$ and $\mathrm{Cl}-$, thus causing ionic imbalance. Due to increased concentration of ions, water accumulates inside cell and it eventually leads to cell death. NKCC1 activity is stimulated by activation of ionotropic and metabotropic glutamate receptors. Thus excitotoxicity caused due to excessive $\mathrm{Cl}-$ and $\mathrm{Na}+$ ions can be averted by either removing these ions from extracellular space or by blocking glutamate receptors [15].

Intracellular calcium is maintained at low concentrations $(\mu \mathrm{M})$ as compared to extracellular calcium. Endoplasmic reticulum and mitochondria constitute important $\mathrm{Ca} 2+$ stores inside cell. $\mathrm{Ca} 2+$ homeostasis of cell is disturbed when glutamate receptor is hyperstimulated. Glutamate ionic receptors assist entry of cations like $\mathrm{Na}+$ and $\mathrm{Ca} 2+$. Increased $\mathrm{Na}+$ concentration inside cell depolarizes cell membrane resulting in opening of voltage-gated $\mathrm{Ca} 2+$ channels, which further increase intracellular calcium level [16]. Higher level of intracellular calcium activates various enzymes like protein kinase II, phospholipases, proteases, nitric oxide synthase and ornithine decarboxylase. These enzymes have different consequences on cell. Activated phospholipase A2 persuades the genesis of arachidonic acid and platelet-activating factor (PAF, 1-O-alkyl-2-acyl-sn-3phosphocholine). PAF induces glutamate release and arachidonic acid impairs reuptake of glutamate from synaptic cleft, resulting in hyperstimulation of glutamate receptor and neurotoxicity [17]. As a result of impairment in reuptake of glutamate, glutamate receptors are 
further stimulated, enhancing more production of arachidonic acid. High level of arachidonic acid generates ROS, which promote protein degradation, DNA fragmentation, and lipid peroxidation of neuronal cells [18].

Raised cytosolic $\mathrm{Ca} 2+$ also stimulates nitric oxide synthase, which induces nitric oxide (NO) synthesis. Being thermodynamically unstable NO reacts with other gaseous molecules, anions and ROS to form nitrates, nitrites and peroxynitrites. Nitric oxide and superoxide radicals combine to form peroxynitrite, which is a highly reactive oxidant and not easily eliminated by antioxidant system. Peroxynitrite has very short half-life of approximately 1-2 seconds and when it degrades it forms many neurotoxic products [19]. Peroxynitrite oxidizes nucleic acids, cytoplasmic proteins and also inhibits mitochondrial respiration by inducing oxidation of electron transport chain (ETC) complexes. Inhibition of ETC complexes leads to formation of ROS, which finally induces apoptosis [20]. NO also evokes apoptosis of neuronal cell by increasing Bax/bcl2 gene expression. The proapoptotic factors, $\mathrm{Bax}$ and $\mathrm{bcl} 2$, promote cytochrome $\mathrm{c}$ release from mitochondria into cytoplasm and activate caspase 3 , stimulating the programmed cell death [21].

\section{Conclusion}

As the mechanism of signaling of glutamate receptor on neuron and platelet is not fully understood, its role in secondary injury after cerebral ischemia remains unclear. Glutamate excitotoxicity caused by platelet aggregates plays significant role in cerebral ischemia. Glutamate ionotropic receptors like NMDAR and AMPAR trigger secondary excitotoxicity by deregulating ionic homeostasis, inhibiting mitochondrial respiration, and generating reactive species of oxygen and nitrogen. Although drugs targeting NMDAR have been developed, they are associated with many deficits and side effects like hallucination, memory loss, ataxia, nightmares and catatonia [22].Thus divulging the signaling mechanism of glutamate ionic receptor system can assist in drug intervention and restoring glutamate mediated excitotoxicity.

\section{References}

1. Sacco RL, Kasner SE, Broderick JP, Caplan LR, Connors JJ, et al. (2013) An updated definition of stroke for the 21st century: a statement for healthcare professionals from the American Heart Association/American Stroke Association. Stroke 44: 2064-2089.

2. Thrift AG, Cadilhac DA, Thayabaranathan T, Howard G, Howard VJ, et al. (2014) Global stroke statistics. Int J Stroke 9: 6-18.

3. Pandian JD, Sudhan P (2013) Stroke epidemiology and stroke care services in India. J Stroke 15: 128-134.

4. Stahl SM, Meltzer HY (1978) A kinetic and pharmacologic analysis of 5hydroxytryptamine transport by human platelets and platelets storage granules: comparison with central serotonergic neurons. J PharmacolExpTher 118-132.

5. Dreux C, Launay JM (1985) Blood platelets: neuronal model in psychiatric disorders. Encephale 11: 57-64.

6. Gill SS, Pulido OM (2001) Glutamate receptors in peripheral tissues: current knowledge, future research, and implications for toxicology. Toxicol Pathol 29: 208-223.
7. Christophe Goubau, Gunnar M Buyse, Chris Van Geet, Kathleen Freson (2014) The contribution of platelet studies to the understanding of disease mechanism in complex and monogenetic neurological disorders. Developmental Medicine and Child Neurology 56: 724-31.

8. Sonkar VK, Kulkarni PP, Dash D (2014) Amyloid $\hat{I}^{2}$ peptide stimulates platelet activation through RhoA-dependent modulation of actomyosin organization. FASEB J 28: 1819-1829.

9. Traynelis SF, Wollmuth LP, McBain CJ, Menniti FS, Vance KM, et al (2010) Glutamate receptor ion channels: structure, regulation, and function. Pharmacol Rev 62: 405-496.

10. Bell JD, Park E, Ai J, Baker AJ (2009) PICK1-mediated GluR2 endocytosis contributes to cellular injury after neuronal trauma. Cell Death Differ 16: 1665-1680.

11. Maggie L KalevZylinska, Taryn N Green, Marie Christine Morel Kopp, Paul P Sun, Young Eun Park, et al. (2014) N-methyl-D-aspartate receptors amplify activation and aggregation of human platelets. Thrombosis Research 133: 837-847.

12. Tremolizzo L, DiFrancesco JC, Rodriguez Menendez V, Sirtori E, Longoni M, et al. (2006) Human platelets express the synaptic markers VGLUT1 and 2 and release glutamate following aggregation. Neuroscience letters 404: 262-265.

13. Joshua D Bell, Theresa currier Thomas, Elliot Lass, Jinglu Ai, Hoyee Wan, et al. (2014) Platelet mediated changes to neuronal glutamate receptor expression at sites of microthrombosis following experimental subarachnoid haemorrhage. J Neurosurg 121: 1424-1431.

14. Germanó A, d'Avella D, Cicciarello R, Hayes RL, Tomasello F (1992) Blood-brain barrier permeability changes after experimental subarachnoid hemorrhage. Neurosurgery 30: 882-886.

15. Beck J, Lenart B, Kintner DB, Sun D (2003) Na-K-Cl cotransporter contributes to glutamate-mediated excitotoxicity. J Neurosci 23: 5061-5068.

16. Arundine M, Tymianski M (2003) Molecular mechanisms of calciumdependent neurodegeneration in excitotoxicity. Cell Calcium 34: 325-337.

17. Bazan NG (1998) The neuromessenger platelet-activating factor in plasticity and neurodegeneration. Prog Brain Res 118: 281-291.

18. Caro AA, Cederbaum AI (2007) Role of intracellular calcium and phospholipase A2 in arachidonic acid-induced toxicity in liver cells overexpressing CYP2E1. Arch Biochem Biophys 457: 252-263.

19. Zhao Feng Peng, Minghui Jessica Chen, Jayapal Manikandan (2012) Multifaceted role of nitric oxide in an in vitro mouse neuronal model: Transcriptomic profiling defines the temporal recruitment of death signaling cascade. Journal of Cellular and Molecular Medicine. 16: 41-48.

20. Brown GC, Bal-Price A (2003) Inflammatory neurodegeneration mediated by nitric oxide, glutamate, and mitochondria. Mol Neurobiol 27: 325-355.

21. Chae IH, Park KW, Kim HS, Oh BH (2004) Nitric oxide-induced apoptosis is mediated by $\mathrm{Bax} / \mathrm{Bcl}-2$ gene expression, transition of cytochrome $\mathrm{c}$, and activation of caspase- 3 in rat vascular smooth muscle cells. Clin Chim Acta 341: 83-91.

22. Stuart A Lipton (2004) Failure and successes of NMDA Receptor Antagonists: Molecular basis for the use of open-channel blockers like memantine in the treatment of acute and chronic neurologic insult. The Journal of the American Society for Experimental NeuroTherapeutics 1: 101-110. 